\title{
3-D ionization structure (in stereoscopic view) of planetary nebulae: the case of NGC 1501
}

\author{
R. Ragazzoni ${ }^{1}$, E. Cappellaro ${ }^{1}$, S. Benetti ${ }^{1}$, M. Turatto ${ }^{1}$, and F. Sabbadin ${ }^{1}$ \\ Osservatorio Astronomico di Padova, vicolo dell'Osservatorio 5, 35122 Padova, Italy
}

Received 9 January 2001 / Accepted 29 January 2001

\begin{abstract}
Long-slit echellograms of the high excitation planetary nebula NGC 1501, reduced according to the methodology developed by Sabbadin et al. (2000a,b), allowed us to obtain the "true" distribution of the ionized gas in the eight nebular slices covered by the spectroscopic slit. A 3-D rendering procedure is described and applied, which assembles the tomographic maps and rebuilds the spatial structure. The images of NGC 1501, as seen in 12 directions separated by $15^{\circ}$, form a series of stereoscopic pairs giving surprising $3-\mathrm{D}$ views in as many directions. The main nebula consists of an almost oblate ellipsoid of moderate ellipticity $(a \simeq 44$ arcsec, $a / b \simeq 1.02, a / c \simeq 1.11$ ), brighter in the equatorial belt, deformed by several bumps, and embedded in a quite homogeneous, inwards extended cocoon. Some reliability tests are applied to the rebuilt nebula; the radial matter profile, the small scale density fluctuations and the 2-D (morphology) - 3-D (structure) correlation are presented and analysed. The wide applications of the 3-D reconstruction to the morphology, physical conditions, ionization parameters and evolutionary status of expanding nebulae in general (planetary nebulae, nova and supernova remnants, shells around Population I Wolf-Rayet stars, nebulae ejected by symbiotic stars, bubbles surrounding early spectral type main sequence stars etc.) are introduced.
\end{abstract}

Key words. planetary nebulae: individual: NGC 1501 - ISM: kinematics and dynamics

\section{Introduction}

The planetary nebula (PN) phase (common to most of intermediate and low mass stars in their late evolution; Iben 1984; Vassiliadis \& Wood 1993; Schönberner 1997) is characterized by an extreme variety of morphologies: disks, butterflies, point-symmetric or multiple shells, hourglasses etc. (Greig 1972; Balick 1987; Schwarz et al. 1992; Manchado et al. 1996).

Different mechanisms, acting before, during and after the nebula ejection, can contribute to generating the observed shapes. These include the mass-loss history, stellar rotation and/or precession, magnetic fields, duplicity of the central star, wind interactions, hydrodynamical processes, ionization and recombination (Tylenda 1986; Morris 1987; Pascoli 1992; Dwarkadas \& Balick 1998; Garcia-Segura et al. 1999).

In order to disentangle the physical processes forming and shaping a PN, the first - and most difficult - observational step is the nebula de-projection, i.e. the recovery of the ionized gas 3-D structure. In this paper, which is the result of a long-standing effort (Sabbadin 1984; Sabbadin

Send offprint requests to: F. Sabbadin,

e-mail: sabbadin@pd.astro.it et al. 1985, 1987, 2000a, 2000b), we obtain the spatial distribution of the PN matter by means of high resolution spectroscopy.

As a test case we selected NGC 1501 for which we have already presented a partial tomographic analysis (Sabbadin et al. 2000b, hereafter P1). In P1, long-slit echellograms at four position angles gave the radial density profile, the mean electron temperature and turbulence and a rough 3-D model. The accurate spatial reconstruction was deferred to a more complete spectroscopic coverage.

Recently we have observed NGC 1501 at four additional position angles and developed a rendering procedure which integrates the tomographic slices and rebuilds the detailed spatial distribution of the ionized gas.

This work, in many respect the logical continuation of P1, is structured as follows: the nebula and the observational material are introduced in Sect. 2; in Sect. 3 we illustrate the reduction procedure, in particular, how to derive the electron density profile from the $\mathrm{H} \alpha$ flux of the zero-velocity pixel column; Sect. 4 contains the tomographic maps and Sect. 5 describes and analyses the spatial model; a general discussion is presented in Sect. 6 and conclusions are drawn in Sect. 7. 


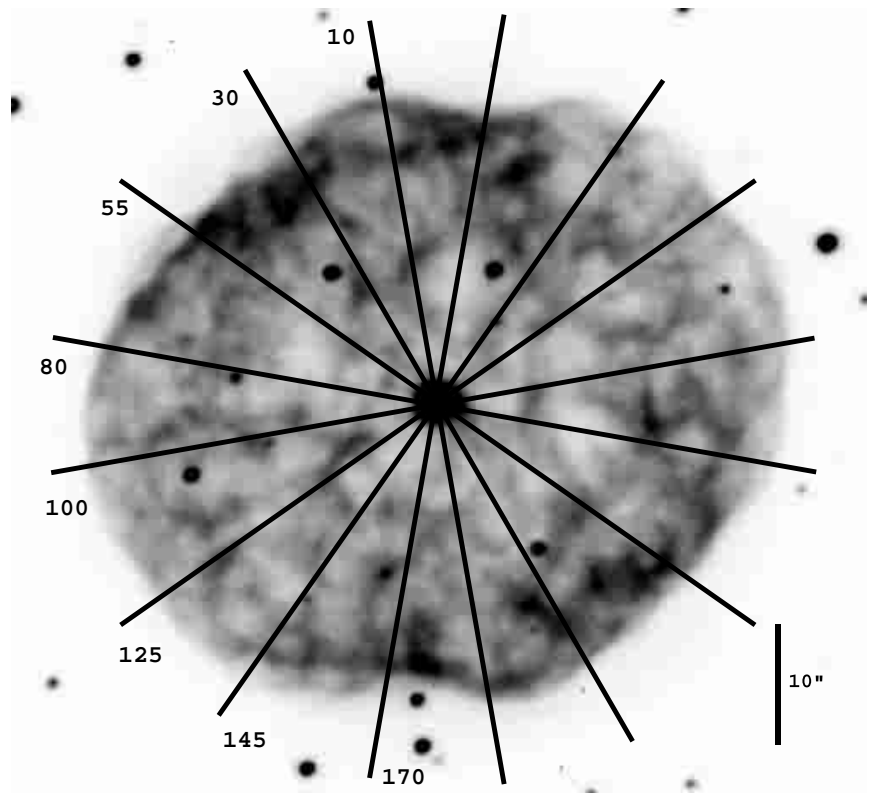

Fig. 1. Spectroscopic slit positions and position angles, superimposed on a broad-band $R$ frame of NGC 1501 taken with the $3.58 \mathrm{~m}$ Italian National Telescope (TNG). The seeing was $F W H M=0.76$ arcsec. North is up and East is to the left

\section{The nebula and the observational material}

The high excitation PN NGC 1501 (PNG144.5+06.5, Acker et al. 1992) is "a mass of irregular condensations, with some big holes" (Minkowski 1968) "bearing a resemblance to the convolutions of the brain" (Pease 1917).

The literature-based data for the whole system (nebula + exciting star) are summarized in Table 1.

$\lambda \lambda 4500-8000 \AA$, spatially resolved spectra of NGC 1501 (including flat field, Th-Ar calibration and spectrophotometric standard) were secured with the Echelle spectrograph attached to the Cassegrain focus of the $1.82 \mathrm{~m}$ Asiago (Italy) telescope and equipped with a Thompson $1024 \times 1024$ pixels CCD (spatial scale $=0.79 \operatorname{arcsec~pix~}^{-1}$; spectral scale $\left.=9.0 \mathrm{~km} \mathrm{~s}^{-1} \mathrm{pix}^{-1}\right)$.

Eight position angles were covered in two observing runs (the slit positions are shown in Fig. 1 superimposed on a broad-band $\mathrm{R}$ frame of the nebula):

Run 1) $\mathrm{PA}=10^{\circ}, 55^{\circ}, 100^{\circ}$ and $145^{\circ}$ on December 1998 (non photometric nights; seeing 1.4-1.7 arcsec; spectral resolution $13.5 \mathrm{~km} \mathrm{~s}^{-1}$; spectra used in $\mathrm{P} 1$ );

Run 2) $\mathrm{PA}=30^{\circ}, 80^{\circ}, 125^{\circ}$ and $170^{\circ}$ on December 1999 (photometric nights; seeing 1.2-1.4 arcsec; spectral resolution $12.0 \mathrm{~km} \mathrm{~s}^{-1}$ ).

In all cases the exposure time was $1800 \mathrm{~s}$.

A detailed description of the spectroscopic characteristics of NGC 1501 is given in P1. In short: it is a high ionization, density bounded PN; stratification effects are negligible; the position-velocity maps can be fitted by a triaxial ellipsoid deformed by several hemispheric bubbles protruding from the central figure; the main emission occurs in a narrow shell whose density profile is steep outwards and flatter inwards.
We believe that a general observational note is appropriate here, concerning the common employment by most authors of an interference filter when obtaining echelle spectra of PNe (to isolate a single order containing one or more interesting emissions, like $\mathrm{H} \alpha+[\mathrm{NII}]$, and to avoid the superposition of orders). No filter was used in our observations, for the following reasons:

- PNe present a discrete spectrum and the superposition of lines belonging to different orders is quite improbable (even with a slit covering several orders);

- the introduction of an interference filter, reducing the spectral range from several hundred $\AA$ to a few dozen $\AA$, drastically limits the number of usable emissions per frame;

- a careful reduction procedure allows us to take into account all the possible sources of inaccuracy (line curvature, order tilting, optical distorsions, spectral sensitivity, CCD cosmetics) over the whole frame, thus obtaining the flux and wavelength calibrated, spatially resolved structure of all emissions present in the echellogram (some dozens in the case of the brightest PNe, representing the main ionic species).

\section{Reduction procedure: the radial electron density $\left(N_{\mathrm{e}}\right)$ profile from the $\mathrm{H} \alpha$ absolute flux in the zero-velocity pixel column}

The spectral reduction process was the same as described in P1, but for the absolute calibration of the radial $N_{\mathrm{e}}$ profile. Due to night sky variability, in P1 we were forced to obtain the electron density of NGC 1501 by comparison with NGC 40 (both nebulae observed on the same nights and with the same instrumental setup). This was unnecessary for "run 2", since the sky was photometric and the proper nebular flux calibration was performed using the spectrophotometric standard. Thus, the starting point is the classical expression

$$
4 \pi D^{2} F(\mathrm{H} \alpha)=h \nu_{3,2} \int_{0}^{R} \alpha_{3,2} N\left(\mathrm{H}^{+}\right) N_{\mathrm{e}} \epsilon 4 \pi r^{2} \mathrm{~d} r
$$

where:

$D$ is the distance, $F(\mathrm{H} \alpha)$ is the $\mathrm{H} \alpha$ flux corrected for extinction, $\nu_{3,2}$ is the $\mathrm{H} \alpha$ frequency, $\alpha_{3,2}$ is the $\mathrm{H} \alpha$ recombination coefficient, $N\left(\mathrm{H}^{+}\right)$is the ionized hydrogen density and $\epsilon$ is the filling factor, representing the fraction of the nebular volume which is filled by matter at density $N_{\mathrm{e}}$.

We restrict the integration to the portion of the nebula identified by the zero-velocity pixel column (corresponding to the gas which is expanding perpendicularly to the line of sight).

The H $\alpha$ flux $\left(F_{l}\right)$ in each pixel of the zero-velocity column, corrected for interstellar absorption and instrument resolution (for details see Sabbadin et al. 2000a), is given by:

$$
4 \pi D^{2} F_{l}=4 \pi j_{\mathrm{H} \alpha} N\left(\mathrm{H}^{+}\right) N_{\mathrm{e}} V_{l} \epsilon_{l} \quad\left[\mathrm{erg} \mathrm{s}^{-1}\right]
$$


Table 1. Basic data of NGC 1501 taken from the literature

\begin{tabular}{|c|c|}
\hline \multicolumn{2}{|r|}{ NEBULA } \\
\hline coordinates $(2000.0)$ & $\alpha: 04^{\mathrm{h}} 06^{\mathrm{m}} 59.7^{\mathrm{s}} \delta:+60^{\circ} 55^{\prime} 14^{\prime \prime}$ \\
\hline apparent size [arcsec] & $56 \times 48($ Curtis 1918); 68 faint, roundish halo $(\mathrm{P} 1)$ \\
\hline $\log F\left(\mathrm{H}_{\beta}\right)\left[\mathrm{mW} \times \mathrm{m}^{-2}\right]$ & -11.28 (Collins et al. 1961); -11.20 (Acker et al. 1991) \\
\hline$c(\mathrm{H} \beta)$ & 0.96 (Kaler 1976); 1.1 (Stanghellini et al. 1994); 1.11 (Ciardullo et al. 1999); 1.05 (P1) \\
\hline excitation class & 8 peculiar (Page 1942) \\
\hline individual distance $[\mathrm{Kpc}]$ & $\begin{array}{l}2.0 \text { (Acker 1978); } 1.4 \text { (Pottasch 1983); } 1.2 \text { (Sabbadin 1986) } \\
\text { (all based on the average extinction in the galactic disk) }\end{array}$ \\
\hline statistical distance $[\mathrm{Kpc}]$ & $\begin{array}{l}1.45 \text { (O’Dell 1962); } 1.20 \text { (Cahn \& Kaler 1971); } 1.78 \text { (Cudworth 1974); } 1.16 \text { (Cahn 1976); } \\
1.16 \text { (Acker 1978); 1.10 (Daub 1982); } 1.10 \text { (Maciel 1984); 0.90 (Amnuel et al. 1984); } \\
1.50 \text { (Sabbadin 1986); } 1.16 \text { (Cahn et al. 1992); } 1.31 \text { (Van de Steene \& Zijlstra 1994); } \\
1.21-1.75 \text { (Zhang 1995) }\end{array}$ \\
\hline adopted distance $[\mathrm{Kpc}]$ & 1.30 \\
\hline mean linear radius $[\mathrm{pc}]$ & 0.164 \\
\hline expansion velocity $\left[\mathrm{km} \mathrm{s}^{-1}\right]$ & $\begin{array}{l}39 \text { in [OIII] (Robinson et al. 1982); } 38 \text { in [OIII] and HI (Sabbadin \& Hamzaoglu 1982); } \\
40 \text { in HeII, [OIII], HI and [NII] (Neiner et al. 2000); } 38 \text { to } 55 \text { in [OIII] and HI (P1) }\end{array}$ \\
\hline turbulence $\left[\mathrm{km} \mathrm{s}^{-1}\right]$ & 10 (Neiner et al. 2000); 18 (P1) \\
\hline structure & $\begin{array}{l}\text { prolate spheroid of moderate ellipticity (Sabbadin \& Hamzaoglu 1982); } \\
\text { thin ellipsoid deformed by a pair of large lobes along both the major } \\
\text { and the intermediate axes and by a heap of bumps spread over the whole } \\
\text { nebular surface (P1) }\end{array}$ \\
\hline \multicolumn{2}{|r|}{ CENTRAL STAR } \\
\hline$m_{V}$ & 14.23 (Tylenda et al. 1991); 14.36 (Ciardullo et al. 1999) \\
\hline spectrum & WC6 (Swings \& Swensson 1942); WC-OVI (Aller 1976); WC4 (Tylenda et al. 1993) \\
\hline $\log T_{\text {star }}$ & $\begin{array}{l}4.98\left(\mathrm{TZ}_{\mathrm{HeII}} ; \text { Sabbadin } 1986\right) ; 4.91\left(T \mathrm{Z}_{\mathrm{HeII}} ; \text { Stanghellini et al. } 1994\right) \\
5.13 \text { (atmosphere model; Koesterke \& Hamann } 1997)\end{array}$ \\
\hline mass loss rate $\left[M_{\odot} \mathrm{yr}^{-1}\right]$ & $5.210^{-7}$ (Koesterke \& Hamann 1997) \\
\hline $\begin{array}{l}\text { terminal wind velocities }\left[\mathrm{km} \mathrm{s}^{-1}\right] \\
\text { note }\end{array}$ & $\begin{array}{l}1800 \text { (Koesterke \& Hamann 1997); } 3260 \text { to } 3460 \text { (Feibelman 1998) } \\
\text { the star is a nonradial } g \text {-mode pulsator with periods ranging from } 5200 \mathrm{~s} \text { to } 1154 \mathrm{~s} \\
\text { (Bond et al. 1998; Ciardullo \& Bond 1998) }\end{array}$ \\
\hline
\end{tabular}

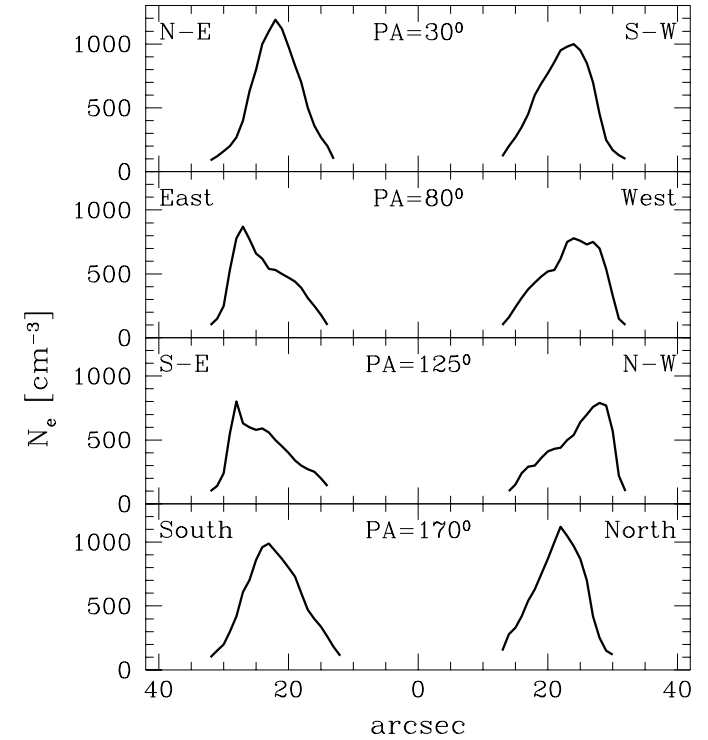

Fig. 2. Radial electron density profiles at the four position angles of "run 2", obtained from the absolute flux in the $\mathrm{H} \alpha$ zero-velocity pixel columns where:

- $j_{\mathrm{H} \alpha}$ is the $\mathrm{H} \alpha$ emission coefficient for the case B of Baker \& Menzel (1938); for typical nebular densities its dependence on $T_{\mathrm{e}}$ is obtained by interpolation of Brocklehurst (1971) data: $4 \pi j_{\mathrm{H} \alpha}=h \nu_{3,2} \alpha_{3,2}=$ $0.2010^{-20} T_{\mathrm{e}}^{-0.94}$;

- $V_{l}$ is the "local nebular volume" defined as $V_{l}=A \times \Delta r$, $A$ being the pixel area and $\Delta r=R \times\left(\Delta V / V_{\exp }\right)$ the radial thickness of the zero-velocity pixel column;

- $\epsilon_{l}$ is the "local filling factor", indicating the fraction of the local nebular volume, $V_{l}$, which is actually filled by matter with density $N_{\mathrm{e}}$.

The radial electron density profiles of NGC 1501 derived from Eq. (2) at the four position angles of "run 2" are shown in Fig. 2 (assuming $T_{\mathrm{e}}=11500{ }^{\circ} \mathrm{K}, N_{\mathrm{e}}=$ $1.15 N\left(\mathrm{H}^{+}\right), c(\mathrm{H} \beta)=1.05, D=1.30 \mathrm{Kpc}$ and, in first approximation, $\epsilon_{l}=1$ ). Their complex structure confirms (at least qualitatively) the results already obtained in P1; in particular, the main nebula consists of a thin shell presenting large inwards tails whose extent is anti-correlated to the height of the density peak.

A quantitative $N_{\text {erun1 }}-N_{\text {erun2 }}$ comparison can be performed only at the intersection of the eight slit positions, 
i.e. at the apparent stellar position; this needs the accurate tomographic reconstruction illustrated in the next section.

\section{Tomography}

The tomographic analysis of PNe, originally introduced and applied to plate spectra by Sabbadin (1984) and Sabbadin et al. $(1985,1987)$, has been recently extended and refined by Sabbadin et al. (2000a, b). Its application to the $\mathrm{H} \alpha$ emission of "run 2" spectra allows us to derive the electron density maps shown in Fig. 3. Following P1, we adopted a linear distance-velocity relation, an electron temperature of $11500{ }^{\circ} \mathrm{K}$, a turbulence of $18 \mathrm{~km} \mathrm{~s}^{-1}$ and a radius of the shell peak at the position of the central star (in radial direction) of 20 arcsec.

The maps of Fig. 3, combined with the similar maps of Fig. 5 of P1, give the spatial $N_{\mathrm{e}}$ distribution in the eight nebular slices covered by the spectroscopic slit. NGC 1501 being a high ionization, density bounded $\mathrm{PN}$, the $N\left(\mathrm{H}^{+}\right)$ and $N\left(\mathrm{O}^{++}\right)$tomographic maps coincide with the $N_{\mathrm{e}}$ ones (but for a scaling factor of 1.15 and $3.810^{3}$, respectively; see P1).

The comparison of the electron densities in the nebular regions common to the eight position angles (i.e. at the apparent stellar position) gives a very satisfactory agreement (to within $\pm 3 \%$ ) between $N_{\text {erun1 }}$ and $N_{\text {erun2 }}$, confirming the validity of the "escamotage procedure" adopted in P1. Our satisfaction is mitigated by the consideration that, in any case, the inaccuracy in the absolute $N_{\mathrm{e}}$ calibration based on Eq. (2) is of the order of $10 \%$, mainly due to the uncertainty in the distance of the nebula.

The only direct $N_{\mathrm{e}}$ determination in NGC 1501 using diagnostic line ratios dates from Aller \& Epps (1976). These authors analysed at low spectral resolution a small region located at a distance of 20 arcsec from the central star $\left(\right.$ in $\left.\mathrm{PA}=125^{\circ}\right)$ and derived $I(6717) / I(6731)=0.78$, corresponding to $N_{\mathrm{e}}=1200 \mathrm{~cm}^{-3}\left(\right.$ for $\left.T_{\mathrm{e}}=11500{ }^{\circ} \mathrm{K}\right)$. From the tomographic map at $\mathrm{PA}=125^{\circ}$ (Fig. 3) we obtain, at a distance of 20 arcsec in the E-SE sector, density peaks of $950-970 \mathrm{~cm}^{-3}$, i.e. our $N_{\mathrm{e}}(S B)$ (SB stands for "surface brightness") is $20 \%$ lower than the corresponding [SII] electron density reported by Aller \& Epps (1976).

Possible causes of this discrepancy are (in order of importance):

a: small-scale density fluctuations. We adopted a local filling factor $\epsilon_{l}=1$; actually, the surface brightness method (cf. Eq. (2)) gives $N_{\mathrm{e}} \times \epsilon_{l}^{1 / 2}$. On the other hand, the [SII] line intensity ratio measures the true electron density of the brightest (i. e. densest) nebular regions; thus the $N_{\mathrm{e}}(S B) / N_{\mathrm{e}}([\mathrm{SII}])$ ratio would indicate a local filling factor $\epsilon_{l} \simeq 0.66$ in the main shell of NGC 1501;

b: distance inaccuracy. From Eq. (2) we have that $N_{\mathrm{e}}(S B) \propto(1 / D)^{1 / 2} ;$ to obtain $N_{\mathrm{e}}(S B)=N_{\mathrm{e}}([\mathrm{SII}])$ we need $D_{\mathrm{NGC} 1501}=0.83 \mathrm{Kpc}$ (assuming $\epsilon_{l}=1$ ). This value is lower than any of the estimates reported in the literature (see Table 1); c: ionization. NGC 1501 being a high ionization PN, $\mathrm{S}^{+}$ is localized in the outer parts of the densest regions, where the shadowing of the UV stellar flux decreases the gas ionization. Thus, the $\mathrm{H}^{+}$and $\mathrm{S}^{+}$spatial distributions are partially decoupled. The effects of ionization on the $N_{\mathrm{e}}(S B) / N_{\mathrm{e}}([\mathrm{SII}])$ ratio are generally modest; they become important in the case of a radial density profile which is multi-peaked, very asymmetric and strongly variable in direction;

$\mathrm{d}$ : measurement uncertainty connected to the weakness of low ionization emissions. A symptom of the difficulties encountered by Aller \& Epps (1976) comes from the non-detection of the [SII] red doublet at a second position close to the nebular edge ( 25 arcsec from the nucleus, in $\mathrm{PA}=223^{\circ}$ ), which is noticeably brighter than the first one. For this second position (intermediate between our $\mathrm{PA}=30^{\circ}$ and $\mathrm{PA}=55^{\circ}$ ) we derive electron density peaks of $1050-1150 \mathrm{~cm}^{-3}$. Note that these $N_{\mathrm{e}}(S B)$ values are only $10 \%$ higher than those obtained for the first position, indicating that the large flux observed in position 2 is mainly due to projection effects (i.e. thickness of the emitting layer).

Most likely, to explain the $N_{\mathrm{e}}(S B)-N_{\mathrm{e}}([\mathrm{SII}])$ discrepancy, we must look for a suitable combination of all the factors listed above. New, valuable information will come out of the detailed spatial reconstruction of the nebular structure, as performed in the next section.

\section{3-D spatial reconstruction}

\subsection{Methodology}

Each tomographic map of NGC 1501 can be regarded as an image $N_{\mathrm{e}}\left(x, x^{\prime}\right)$, where $x$ is the distance from the plane of the sky crossing the central star and $x^{\prime}$ is measured along the slit. In order to recover the spatial electron density distribution $N_{\mathrm{e}}(x, y, z)$, a suitable reference system is defined: we adopted $x$ pointing toward the Observer, $y$ toward East and $z$ toward North.

A data-cube of a given size $N \times N \times N$ is initialized, filled with the raw data and re-mapped into cylindrical coordinates $(x, r, \alpha)$, where:

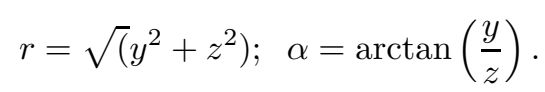

Once we have defined the pixel size $p$, we can fill up the $n$ planes related to the spectrograms as:

$N_{\mathrm{e}}(x, r, \alpha)=N_{\mathrm{ei}}\left(\frac{x}{p}, \frac{x^{\prime}}{p}\right)$.

Interpolation between the $n$ observed planes is made in a linear fashion along the cylindrical coordinates. In other words, the data for $\alpha>\alpha_{i}$ and $\alpha<\alpha_{i+1}$ are given by:

$N_{\mathrm{e}}(x, r, \alpha)=N_{\mathrm{ei}}\left(\frac{x}{p}, \frac{x^{\prime}}{p}\right) \frac{\delta \alpha_{i}^{-}}{\Delta \alpha}+N_{\mathrm{ei}+1}\left(\frac{x}{p}, \frac{x^{\prime}}{p}\right) \frac{\delta \alpha_{i}^{+}}{\Delta \alpha}$

where:

$\delta \alpha_{i}^{-}=\alpha-\alpha_{i}, \delta \alpha_{i}^{+}=\alpha_{i+1}-\alpha$ and $\Delta \alpha=\alpha_{i+1}-\alpha_{i}$. 

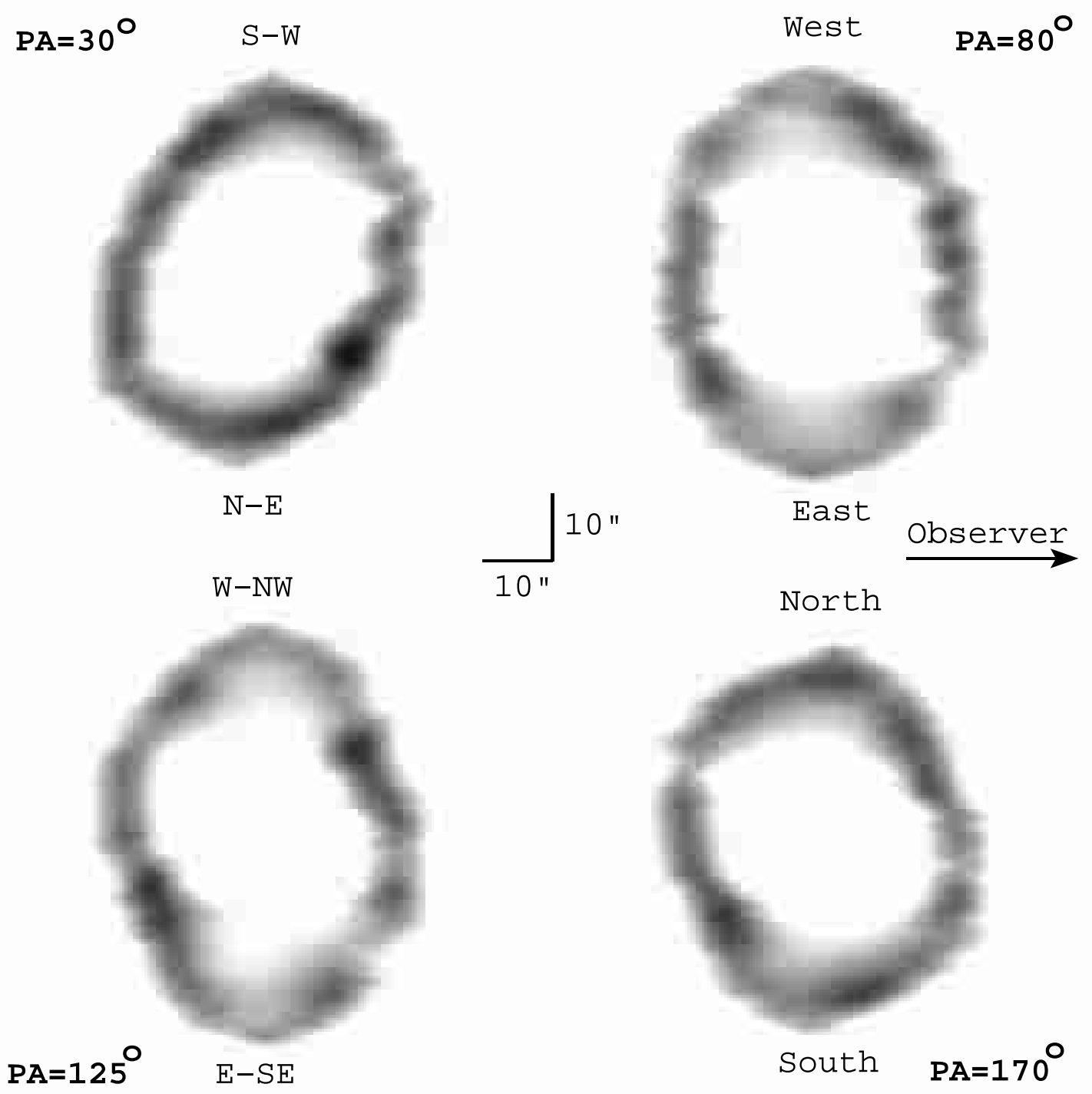

Fig. 3. True electron density structure in the four slices of NGC 1501 covered by the spectroscopic slit during "run 2". The lowest density shown is $N_{\mathrm{e}}=200 \mathrm{~cm}^{-3}$, while the highest one $\left(N_{\mathrm{e}}=1410 \mathrm{~cm}^{-3}\right)$ is reached by the approaching knot in PA $=30^{\circ}$ (N-E sector), at an apparent distance of 11 arcsec from the central star

Finally, one should consider the singularity in $y=z=0$. These points are treated as an average of the neighbouring pixel-data for each plane defined by a given $x$.

In the examples reported here we used $N=200, n=8$ and $p=0.40$ arcsec.

\subsection{Results}

A problem arises when presenting the $N_{\mathrm{e}}$ data-cube of NGC 1501: how can we render the 3-D on the page? The adopted solution is illustrated in the following figures.

Figure 4 shows the opaque reconstruction of the densest nebular regions $\left(N_{\mathrm{e}}>900 \mathrm{~cm}^{-3}\right)$ as seen from $12 \mathrm{di}-$ rections, separated by $15^{\circ}$.

Figure 5 is the same as Fig. 4, but for the weakest components $\left(N_{\mathrm{e}}>300 \mathrm{~cm}^{-3}\right)$; at even lower densities the roundish halo appears.
Finally, Fig. 6 represents the optical appearance of the rebuilt-nebula seen from the same 12 directions of Figs. 4 and 5.

In these figures the line of view is identified by the zenith angle $(\theta)$ and the azimuthal angle $(\psi)$, corresponding to a rotation through the first two Euler angles. Thus, for example, the upper-right image represents the rebuilt nebula as seen from the Earth (i.e. from $(0,0)$ ).

Let's consider Figs. 4 and 5. Since the line of view of two adjacent images differs by $15^{\circ}$, each horizontal couple constitutes a stereoscopic pair, allowing the reader to enjoy 12 surprising 3-D views of the nebula in as many directions (instructions are given in the caption of Fig. 4). The stereoscopic view is more difficult in Fig. 6, because of the diffuse, smooth nebular appearance.

Hereafter we will call "direct stereoscopy" the 3-D view just described; in Sect. 6.3 an alternative method, 


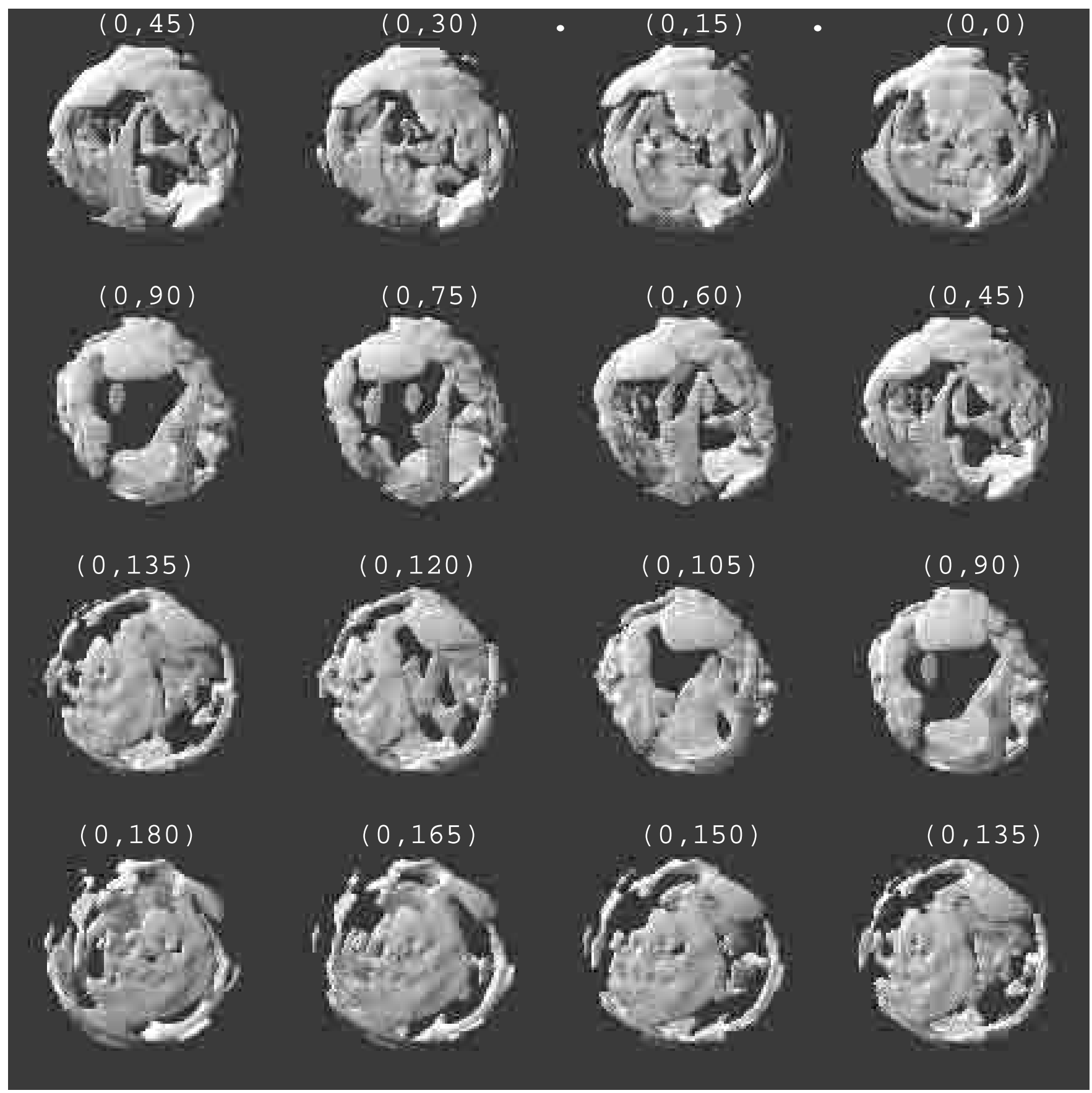

Fig. 4. Opaque reconstruction of the densest regions of NGC $1501\left(N_{\mathrm{e}}>900 \mathrm{~cm}^{-3}\right)$ as seen from 12 directions separated by $15^{\circ}$. The line of view is identified by $(\theta, \psi)$, where $\theta$ is the zenith angle and $\psi$ the azimuthal angle, representing a rotation through the first two Euler angles; the upper-right image is the rebuilt-nebula as seen from the Earth, i.e. from $(0,0)$. Each horizontal couple constitutes a "direct" stereoscopic pair, allowing the reader to have $123-\mathrm{D}$ views of the nebula in as many directions (all together covering a straight angle). Instructions for beginners: to obtain the three-dimensional vision, look at a distant object and slowly insert the figure in the field of view (always maintaining your eyes parallel). Alternatively, you can use the two small dots in the upper part of the figure as follows: approach the page till the two dots merge (they appear out of focus); then recede very slowly (always maintaining the two dots superimposed) till the image appears in focus. A suggestion: be patient. The difficulties encountered in the beginning soon disappear and you will have exciting three-dimensional views of the nebula

"swapped stereoscopy", will be introduced and compared with the "direct" one.

For reasons of space, only twelve low resolution nebular structures (at two density cuts) are presented, and as many projections. The complete data-cube can be directly requested from the authors. Moreover, some movies of NGC 1501 (+ other pleasantries) will be available soon from a dedicated WEB page. 


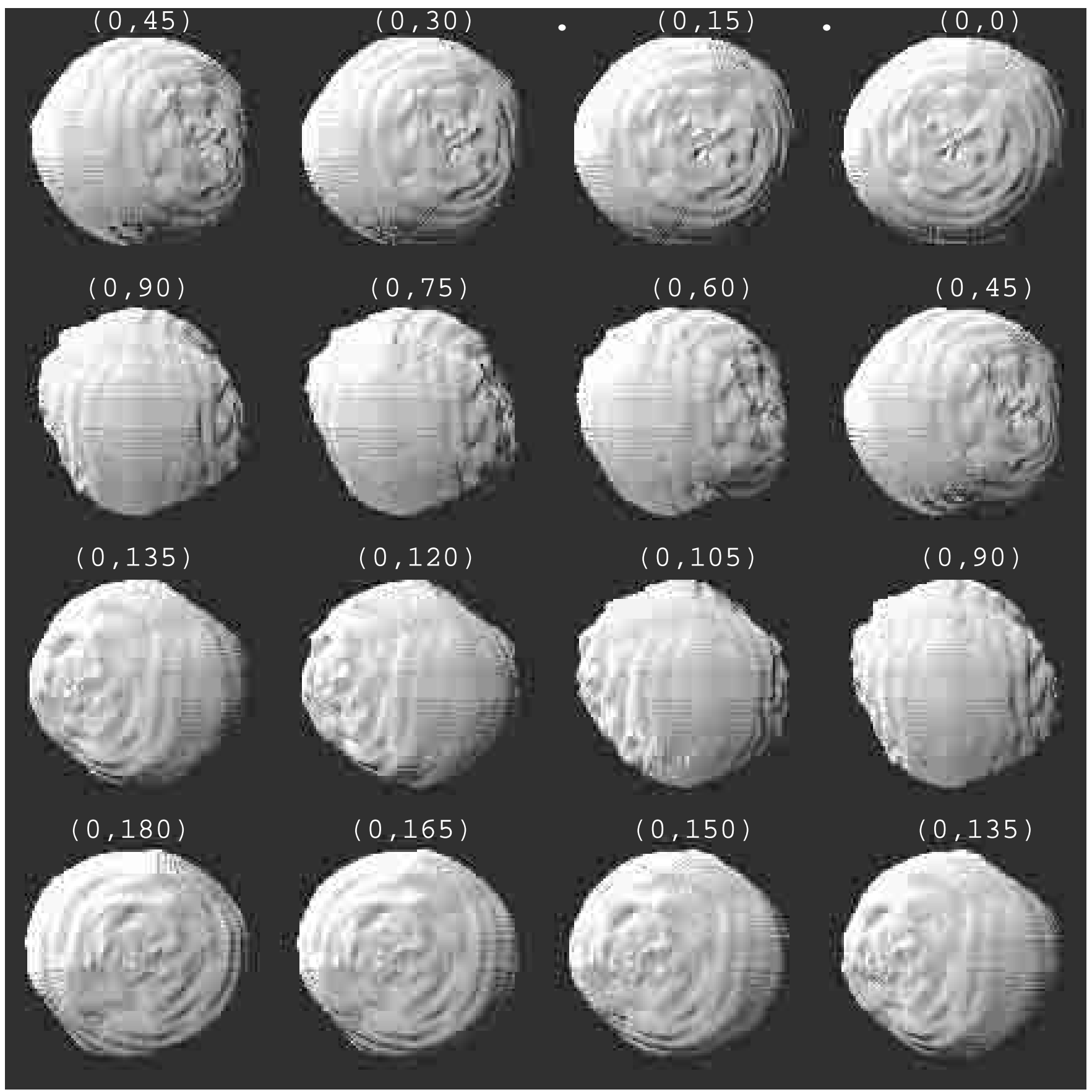

Fig. 5. Same as Fig. 4, but for the weakest components $\left(N_{\mathrm{e}}>300 \mathrm{~cm}^{-3}\right)$ of NGC 1501. To enjoy the stereoscopic view of the nebula in 12 directions, read the instructions contained in the caption of Fig. 4

\subsection{Reliability tests}

The first (and most important) consistency test of our reconstruction method is based on the comparison of the optical appearance of the true- and the rebuilt-nebula, when reduced to the same angular resolution.

To be noticed that the spatial resolution of NGC 1501rebuilt is not constant, but anti-correlated to the angular distance from the central star (this occurs because we observed the nebula at radially arranged position angles). Thus, taking into account the slit width, the number of position angles and the nebular angular extension, we have that the spatial resolution of NGC 1501-rebuilt varies from $1.5-2.0$ arcsec (at the central star position) to 3.0-3.5 arcsec (at the nebular edge).

The optical appearance of the true nebula under a seeing of 2.0 and 3.5 arcsec is shown in Fig. 7. The comparison with NGC 1501-rebuilt (Fig. 6 upper-right image) is very satisfactory, both qualitatively and quantitatively. Indeed:

1. all the macro- and micro-characteristics observed in NGC 1501 (general appearance, holes position, blobs and condensations distribution etc.) are faithfully reproduced by the model; 


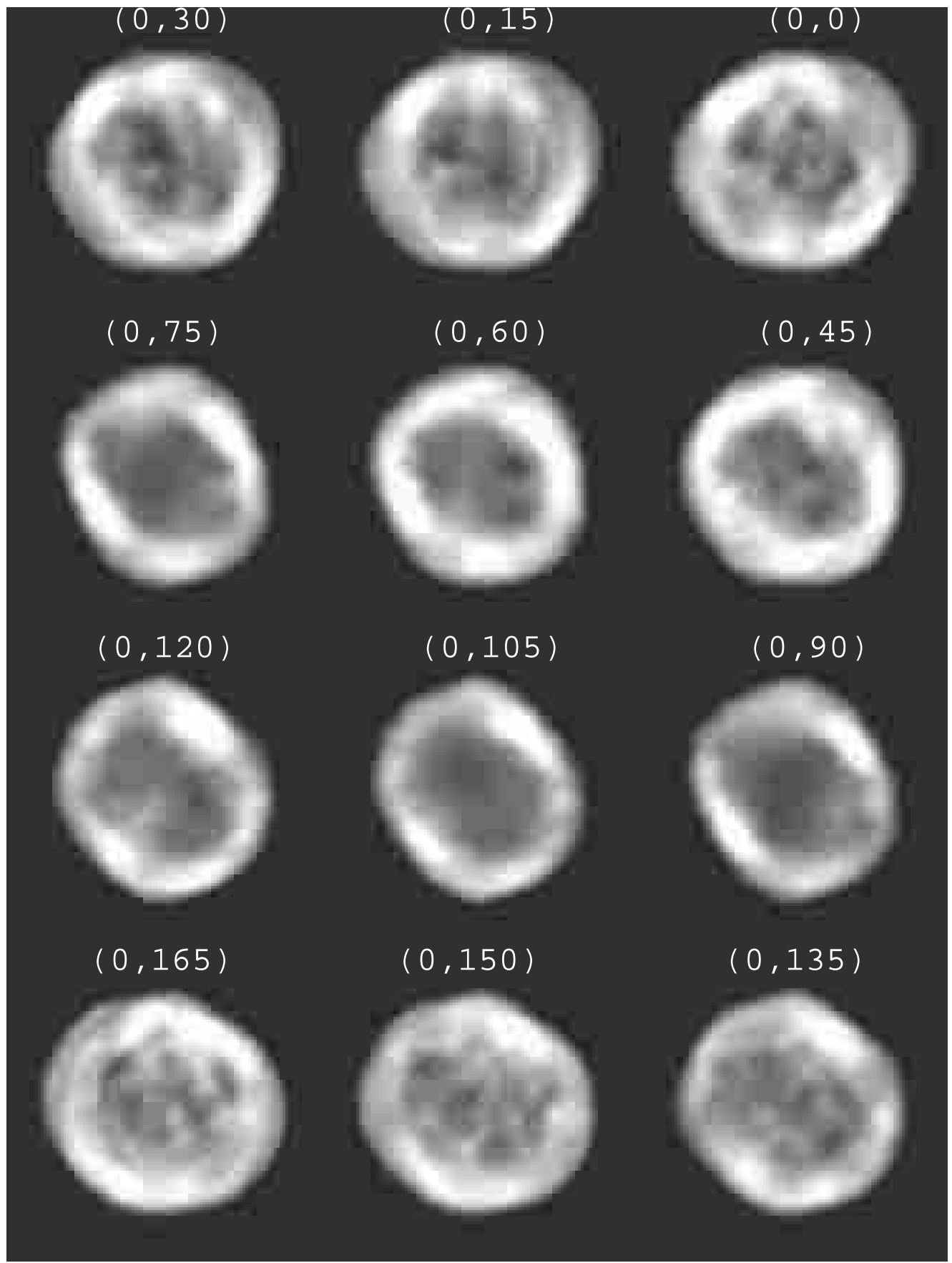

Fig. 6. Optical appearance of NGC 1501-rebuilt from the same 12 directions of Figs. 4 and 5 (and at the same scale). Note that projection $(0, \psi)=\operatorname{projection}\left(0, \psi \pm 180^{\circ}\right)$. The upper-right image corresponds to the rebuilt-nebula as seen from the Earth. In this case the stereoscopic view is quite difficult, because of the smooth, diffuse nebular appearance

2. the surface brightness profiles of the two nebulae agree to within $\pm 10 \%$ along the position angles covered by the spectroscopic slit and to within $\pm 20 \%$ at intermediate directions.

Other checks, e.g. the comparison of the nebular fluxes at different radii and/or different positions, the $N_{\mathrm{e}}$ distribution etc. confirm that our technique adequately recovers the 3-D nebular shape. In conclusion, Figs. 4 to 6 closely represent both the structure and appearance of NGC 1501 rotating around a $\mathrm{N}-\mathrm{S}$ axis centred on the exciting star.

\section{Discussion}

In general, the foregoing spatial reconstruction performed in different emissions (i.e. for various ions) allows us to obtain the detailed 3-D ionization structure of a PN. This is particularly valuable for objects presenting complex morphologies (butterflies, poly-polars, multi-shells etc.) and/or large stratification effects and/or small scale condensations (ansae, knots, FLIERS = fast, low ionization emitting regions, BRETS = bipolar, rotating, episodic jets etc.). 


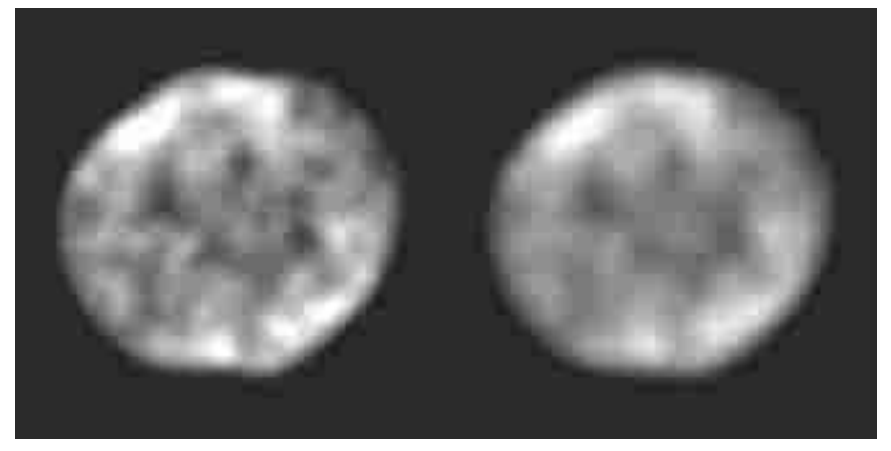

Fig. 7. Optical appearance of NGC 1501 (the true nebula) under a seeing of $2.0 \operatorname{arcsec}$ (left) and $3.5 \operatorname{arcsec}$ (right). We have first removed the stars from the TNG $\mathrm{R}$ frame, and later blurred the image by convolution with a Gaussian having $F W H M=2.0$ arcsec and 3.5 arcsec, respectively. The field and the orientation are as in Fig. 1. You can compare these images of the true nebula with the one of NGC 1501-rebuilt seen from $(0,0)$, i.e. with the upper-right image of Fig. 6 (recall that the spatial resolution of NGC 1501-rebuilt is anti-correlated to the distance from the central star: $1.5-2.0$ arcsec at the stellar position to $3.0-3.5$ arcsec at the nebular edge)

Moreover, photo-ionization models at unprecedented resolution of the whole nebula and of the micro- and macro-structures can be produced by combining the true spatial structure of the expanding gas with the ultraviolet stellar flux.

Finally, the 3-D reconstruction can strongly contribute in solving the PNe "Problem", i.e. the distance: only the detailed knowledge of both the dynamical and physical nebular properties will give the right interpretation of the angular expansion measured in first and second epoch HST and/or radio imagery, thus obtaining precise expansion-parallax distances (Hajian et al. 1993, 1995; Kawamura \& Masson 1996; Terzian 1997).

From all these points of view NGC 1501 is a complete disappointment: it is quite faint (i.e. only the brightest emissions can be analysed) and homogeneous (no knots, jets or ansae) at high excitation and density bounded (stratification of the radiation is negligible).

In spite of this, the 3-D analysis of our "ordinary" $\mathrm{PN}$ is important to introduce the wide potentialities of the spatial reconstruction in studying at small and large scales both the phenomenology and the physical processes connected with expanding nebulae (PNe, nova shells, supernova remnants, nebulae around Population I Wolf-Rayet stars etc.).

\subsection{General 3-D structure of NGC 1501}

NGC 1501 is a high excitation, density bounded PN, and its spatial structure is the same in $N_{\mathrm{e}}, N\left(\mathrm{H}^{+}\right)$and $N\left(\mathrm{O}^{++}\right)$, other than a scaling factor.

Low ionization regions (for example $\mathrm{N}^{+}$, identified by $\lambda 6584 \AA$ of $[\mathrm{NII}])$ are too weak for a detailed 3-D analysis. Probably they are localized in the external parts of the densest knots and condensations, where the shadowing of the ultraviolet stellar flux lowers the nebular ionization.

The results obtained in $\mathrm{P} 1$ (the main body of NGC 1501 is a thin ellipsoid of moderate ellipticity deformed by a pair of large lobes along both the major and intermediate axes and by a multitude of smaller bumps spread on the whole nebular surface, making the general 3-D structure like a boiling, tetra-lobed shell) can now be improved and extended.

The thin, inhomogeneous shell forming the main nebula (Fig. 4) is characterized by a dense, oblique belt definiing the "equatorial" plane, i.e. the plane containing the axes $b$ and $c$ of the central ellipsoid. The maximum density $\left(N_{\mathrm{e}} \simeq 1400 \mathrm{~cm}^{-3}\right.$ for $\left.\epsilon_{l}=1\right)$ is reached in the direction of the minor axis. Only some arcs and knots belonging to the lobes appear in Fig. 4.

A lumpy, quite homogeneous cocoon at low density completely embeds the main shell (Fig. 5). The complex radial structure of this envelope (sharp outwards and broad inwards) cannot be seen here and will be presented and discussed later (Sect. 6.4).

The "boiling" structure of NGC 1501 is clearly visible in Figs. 4 and 5 for the shell's regions with higher spatial resolution, i. e. those projected on - or close to - the central star when the nebula is seen from $(0,0)$.

To highlight the "tetra-lobed" shape, in Fig. 8 we present the optical appearance of NGC 1501 as seen from:

a) $(25,75)$, i.e. along the major axis of the ellipsoid;

b) $(65,-15)$, i.e. along the intermediate axis;

c) $(-25,-15)$, i.e. along the minor axis.

In conclusion: the main body of NGC 1501 is a deformed, almost oblate ellipsoid of moderate ellipticity $(a \simeq$ $44 \mathrm{arcsec}, a / b \simeq 1.02, a / c \simeq 1.11)$, denser in the equatorial belt containing the axes $b$ and $c$. Hydrodynamical processes (possibly Rayleigh-Taylor instabilities and wind interaction) partially swept up the lower density regions of the ellipsoid, generating both the hemispheric caps and the broad inward tail in the radial matter distribution.

The ionized nebular mass $\left(M_{\text {ion }}\right.$, coinciding with the total nebular mass $)$ is $0.15( \pm 0.03) M_{\odot}$ and the dynamical age $5000( \pm 500)$ years.

As noticed in P1, a small, attached halo extending up to 34 arcsec from the central star surrounds the main nebula. The roundish, homogeneous appearance of the halo suggests that it represents photospheric material ejected by the PN progenitor in the late AGB evolution. Moreover, the radial surface brightness profile, steeper than the $r^{-3}$ law expected of a steady-state flow, indicates that the halo corresponds to a phase of "enhanced" stellar mass-loss. Finally, the electron density profile (Fig. 2 of this paper and Fig. 4 of P1) gives a mass-loss rate of $3( \pm 1) 10^{-5} M_{\odot} \mathrm{yr}^{-1}$ and an age of $1.0( \pm 0.2) 10^{4}$ years (assuming $V_{\exp }=20 \mathrm{~km} \mathrm{~s}^{-1}$; see Habing 1996 and references therein).

We claim that the halo represents the vestiges of the "superwind" phase (lasted $5( \pm 1) 10^{3}$ years) which generated the nebula. Most of the gas was later swept up 


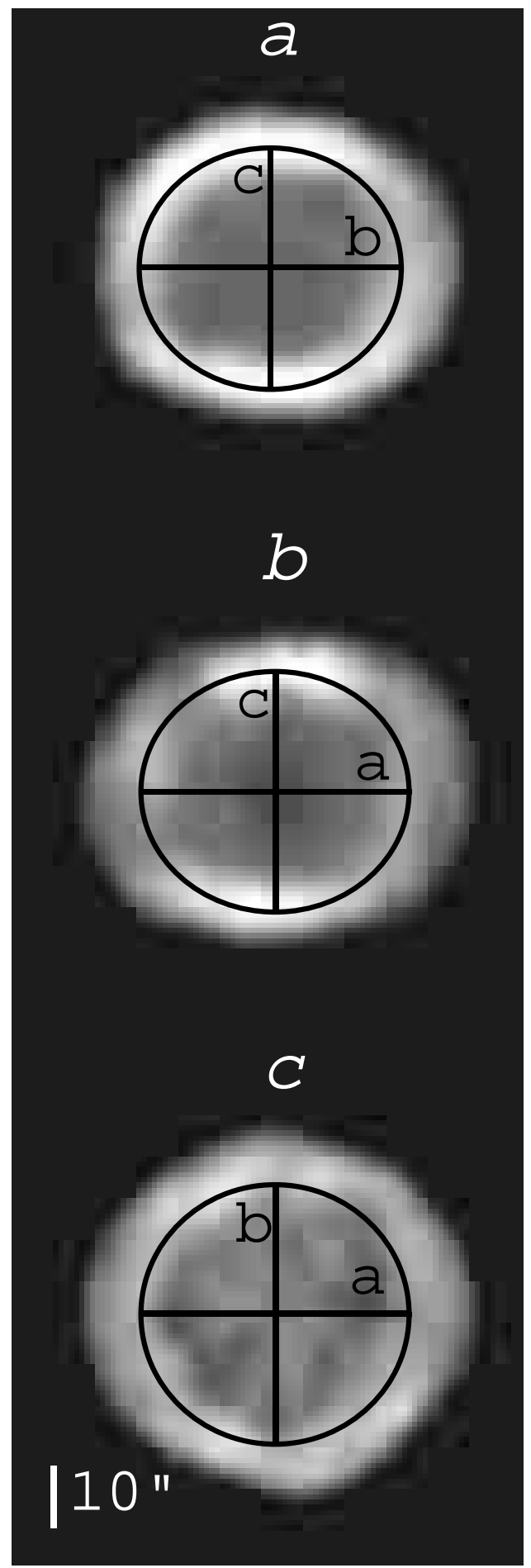

Fig. 8. Optical appearance of NGC 1501-rebuilt seen from a) $(25,75)$ i.e. along the major axis of the ellipsoid; b) from $(65,-15)$ i.e. along the intermediate axis and c) from $(-25,-15)$ i.e. along the minor axis. The thin ellipsoid's section and the axes contained in the plane of each figure are shown

by wind interaction, and was completely ionized by the UV flux of the evolving star, thus producing NGC 1501. Note that the mean mass-loss rate in the superwind phase resulting from the analysis of the main nebula, given by
( $M_{\text {ion }} /$ superwind duration), coincides with the value just obtained for the halo.

At this point a quick comparison with NGC 40 (the first PN tomographically studied in detail, Sabbadin et al. 2000a) can be instructive.

NGC 40 is an optically thick, very low excitation barrel-shaped nebula with thin arcs emerging at both ends of the major axis; it is powered by a luminous WC8 star presenting a large mass-loss rate. According to the "bornagain scenario" proposed by Iben (1984; see also Blöcker 1995 and references therein), the central stars of NGC 40 and NGC 1501 suffered a final thermal pulse in the late post-AGB phase, ejecting the photospheric strata and exposing the $\mathrm{C}$ and $\mathrm{O}$ rich core.

Moreover, both the evolutionary sequence [ $\left.W C_{\text {late }}\right]-$ $\left[W C_{\text {early }}\right]-[W C$-PG 1159] - [PG 1159] (Hamann 1997; Koesterke et al. 1997) and the dynamical ages (3500 \pm 500 yr for NGC 40 and $5000 \pm 500$ yr for NGC 1501) would indicate a earlier evolutionary phase for NGC 40.

In $2000 \pm 500 \mathrm{yr}$ its size should be comparable with the present size of NGC 1501, but the structure will be quite different: a dense (optically thick?) equatorial torus + extended, faint polar caps.

Thus: NGC 40 will most likely evolve into a bipolar (butterfly?) PN, whereas NGC 1501 will keep its ellipsoidal structure, becoming fainter and fainter (in a few thousand years it will resemble A 43 and NGC 7094, two extended, filamentary, high ionization PNe excited by hybrid-PG 1159 type central stars; see Rauch 1999 and Feibelman 2000).

The different evolution of the two nebulae can be tentatively explained by a more massive NGC 40 progenitor, which ejected a higher nebular mass with a larger density gradient between equatorial and polar regions, and created a higher mass central star (with respect to NGC 1501).

Further support for this qualitative scenario comes from a preliminary analysis of a third "born-again" PN at an even earlier evolutionary phase, BD+303639 (the Campbell's star), observed with TNG + SARG in nine position angles at spatial and spectral resolutions of 0.7 arcsec and $\lambda / \Delta \lambda=115000$, respectively.

\subsection{2-D (morphology) - 3-D (structure) correlation}

Because of its fascinating variety and importance in most aspects of the advanced evolution of low and intermediate mass stars, the morphology of PNe has attracted many authors and several efforts have been made to search for a general classification scheme for the "morphological forest" (Greig 1972; Zuckerman \& Aller 1986; Balick 1987; Chu et al. 1987; Balick et al. 1992; Stanghellini et al. 1993; Corradi \& Schwarz 1995; Manchado et al. 1996).

At the same time, a number of hydrodynamical and magnetohydrodynamical simulations were carried out and the theoretical nebulae compared with the observed ones (Mellema 1997; Dwarkadas \& Balick 1998; Garcia- Segura et al. 1999 and references therein). 
Our 3-D reconstruction method, allowing us to derive the detailed spatial structure of each object and to observe it from all possible directions, revolutionizes the approach to the morphological problem of $\mathrm{PNe}$ and opens new prospectives for understanding the 2-D (morphology) - 3-D (structure) relation.

Let's consider Fig. 6, for instance, giving a representative sample of the manifold morphologies of NGC 1501 when changing the line of view.

The optical appearance of the nebula mainly depends on the "latitude", i.e. on the angle of view of the dense, inhomogeneous equatorial belt: at "low latitudes" (from $(0,135)$ to $(0,15)$ in Fig. 6$)$ it is a broad disk presenting inner, amorphous structures. When seen almost poleon (from $(0,60)$ to $(0,105))$, a sharp, oval ring appears, brighter along the minor axis (corresponding to the projection of the $c$ axis of the ellipsoid).

A quick look through the main imagery catalogues (Acker et al. 1992; Schwarz et al. 1992; Manchado et al. 1996; Gorny et al. 1999) indicates that NGC 1501-rebuilt resembles M 3-30 when seen from $(0,15)$, A 53 and NGC 7094 from $(0,30)$, IC 1454 from $(0,45)$, A 73 from $(0,60)$, A 70 from $(0,105)$, NGC 4071 from $(0,120)$ and NGC 6894 from $(0,135)$.

We can add that, seen from other directions (not presented here for reasons of space), NGC 1501 looks like several other nebulae contained in the above-mentioned catalogues.

In short: all the possible morphologies assumed by NGC 1501 when seen from different directions fall in the categories named C (centric PN) by Greig (1972), Def (elliptical disk with filaments) by Zuckerman \& Aller (1986), E (elliptical) by Balick (1987), ES (elliptical with inner filaments) by Stanghellini et al. (1994), E (elliptical) by Corradi \& Schwarz (1995) and Es (elliptical with inner structure) by Manchado et al. (1996). They correspond to quite regular and symmetric shells, rings or disks probably ejected by single, low mass progenitors $\left(1.1 M_{\odot}<M_{\mathrm{MS}}<2.5 M_{\odot}\right)$, excited by low mass central stars $\left(0.56 M_{\odot}<M_{\mathrm{CS}}<0.60 M_{\odot}\right)$ showing a galactic distribution and kinematics typical of the old Disk Population (Peimbert \& Serrano 1980; Corradi \& Schwarz 1995; Maciel \& Quireza 1999 and references therein).

In a certain sense, all this belongs to the past: the understanding of the different mechanisms and physical processes forming and shaping PNe passes through the knowledge of the envelope structures. In their turn, these 3-D structures are usually presumed from a schematic classification of the manifold nebular morphologies. Such a rough cascade process is overcome by our 3-D analysis, which directly provides the accurate spatial distribution (and ionization) of the expanding gas, thus removing any misleading camouflage due to projection.

An example: while it is evident that in no case will NGC 1501 appear as a butterfly or a hourglass, the opposite could occur: when seen at particular directions (e.g. along - or close to - the axis of the lobes), a genuine butterfly or hourglass PN (like NGC 2346 or NGC 650-1) could mimic some NGC 1501 appearances (as suggested for K 4-55 by Guerrero et al. 1996).

This could also be the case for some famous and extensively studied PNe, like NGC 6720. The three-dimensional structure of the Ring Nebula has never been firmly defined, spanning from a complex toroid viewed close to the polar axis (Minkowski \& Osterbrock 1960; Louise 1974; Reay \& Worswick 1977; Balick et al. 1992; Volk \& Leahy 1993; Bryce et al. 1994; Lame \& Pogge 1994) to an oblate or prolate spheroidal or ellipsoidal shell (Atherton et al. 1978; Phillips \& Reay 1980; Kupferman 1983; Masson 1990; Pascoli 1990; Guerrero et al. 1997), to a flat ring (Hua \& Louise 1970) and to a hollow cylinder (Proisy 1974; Bachiller et al. 1989). No doubt the Ring Nebula will be one of our next targets.

\subsection{Zooming in NGC 1501: local filling factor, $\epsilon_{1}$, and density fluctuations in the main shell}

Figures 4,5 and 6 clarify that the big holes characterizing the optical appearance of NGC 1501 represent localized shell regions of lower density. This is even more evident in Fig. 9, showing the "swapped stereoscopic" nebular structure for $N_{\mathrm{e}}>700 \mathrm{~cm}^{-3}$ as seen from $(0,0)$, i.e. from the Earth.

The hole in $\mathrm{PA}=70^{\circ}$ at 11 arcsec from the central star corresponds to a "window" in the approaching nebular gas; through this "window" we observe the receding, dense material in the oblique belt representing the equatorial region of NGC 1501 . The hole is clearly visible also in the following tomographic maps: Fig. 3 at $\mathrm{PA}=80^{\circ}$, East sector and Fig. 5 of $\mathrm{P} 1$ at $\mathrm{PA}=55^{\circ}$, N-E sector.

Since in the hole $N_{\mathrm{e}}$ (receding gas) $/ N_{\mathrm{e}}$ (approaching gas) $>1.6$, we have flux(rec. gas)/flux(app. gas) $>2.5$; i.e. to a first approximation we can neglect the contribution of the approaching material and assume that we are observing only the receding one. In this way we have isolated a well-defined nebular portion, appropriate for study in detail at the maximum spatial resolution (TNG or, better, HST imagery).

Figures $10 \mathrm{a}, \mathrm{b}$ and $\mathrm{c}$ show the flux maps in the $15 \times$ $15 \operatorname{arcsec}^{2}$ nebular region centred on the hole at $11 \operatorname{arcsec}$ from the central star in $\mathrm{PA}=70^{\circ}$, as given by our $\mathrm{TNG}$ broad-band R frame (Fig. 10a), by the same frame after a soft Lucy-Richardson restoration (Fig. 10b), and by HST (Fig. 10c).

These figures stand out intensity fluctuations in the hole by a factor of 2.0, 2.5 and 3.0 for the TNG, TNG + Lucy-Richardson and HST images, respectively, indicating a clear correlation with the angular resolution.

Let's take in the receding portion of the nebular shell observed through the hole:

- the HST value as representative of the small scale flux variations;

- the $N_{\mathrm{e}}$ radial profile derived from the 3-D reconstruction;

- the constancy of the electron temperature. 


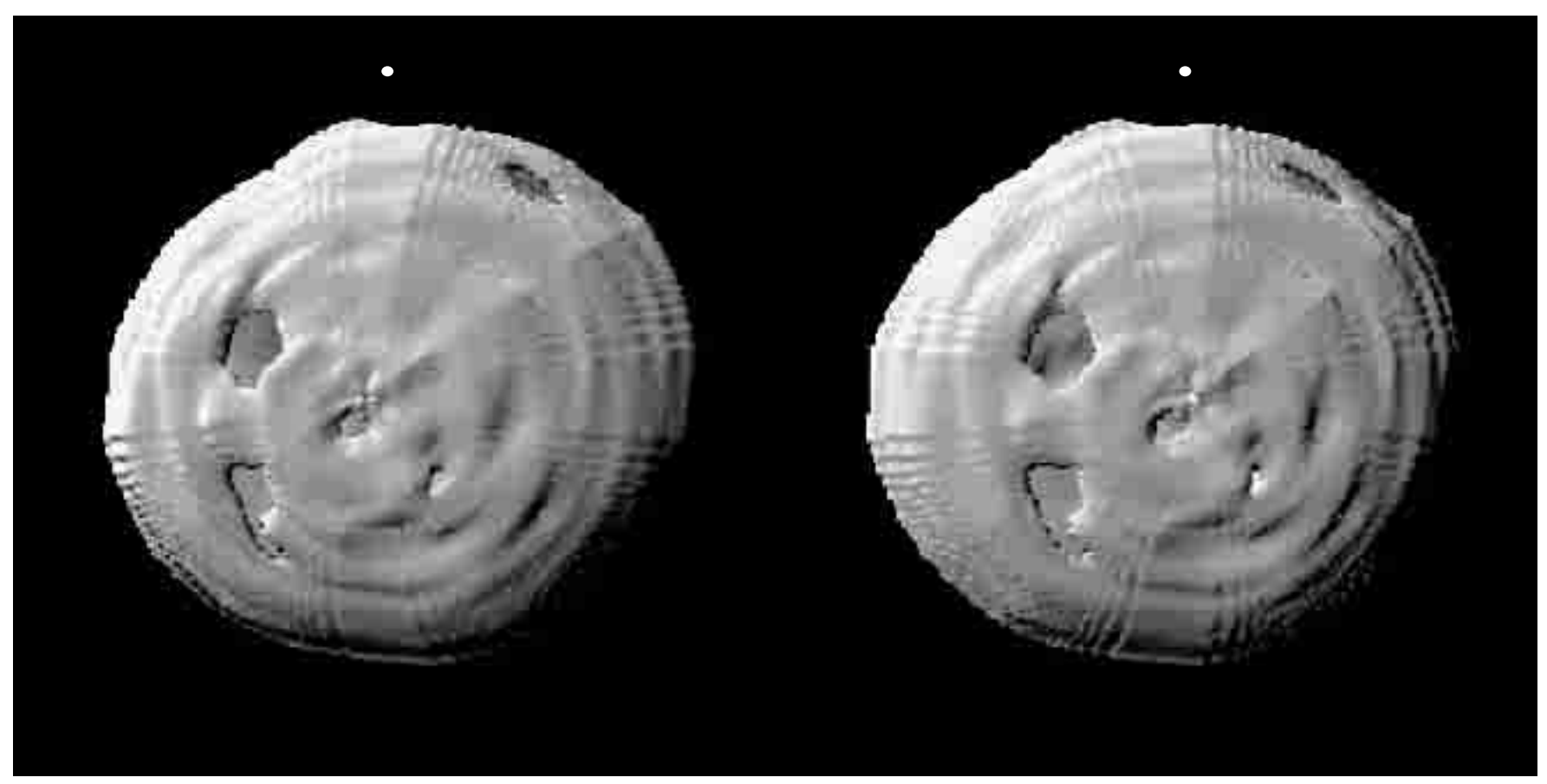

Fig. 9. Opaque reconstruction of NGC 1501 for $N_{\mathrm{e}}>700 \mathrm{~cm}^{-3}$, as seen from $(0,-7$; left) and $(0,7$; right), allowing the reader to have a "swapped stereoscopic vision" of the nebula from $(0,0)$, i.e. from the Earth. To enjoy the 3-D view, cross your eyes till the two dots in the upper part of the figure perfectly coincide (in this way the left eye is forced to observe the right image, and viceversa). "Swapped stereoscopy" presents a great advantage with respect to the "direct" one: the image size can be as large as one wishes, thus achieving a better resolution than the "direct method" (where the centres of two adjacent images must be closer than the pupils separation). The drawback of "swapped stereoscopy" is the prohibitive physical size of a figure containing a large number of images (as in the case of Figs. 4 and 5). Therefore, "direct stereoscopy" is convenient when presenting many pairs. Notice that in both methods a myope has an advantage, since he/she focuses closer. Caveat for beginners: when looking at a swapped stereoscopic pair for a long time, a slight trouble can arise (due to squint). Don't worry, but be careful. The field and the orientation are as in Fig. 1

Under these assumptions we obtain fluctuations of the density peaks of a factor $1.5( \pm 0.2)$.

Moreover, using a black-body spectrum for the exciting star and "normal" chemical abundances for the expanding gas (see Aller \& Czyzak 1983), we have that:

- the electron density peaks given by the [SII] red doublet are 1.10-1.20 larger than $N_{\mathrm{e}}(S B)$;

- due to the incomplete recombination of $\mathrm{S}^{++}$, the $[\mathrm{SII}]$ $6717 / 6731 \AA$ density peaks are 10 to $30 \%$ lower than the true $N_{\mathrm{e}}$ values.

This is valid for a wide range of nebular distances $(1.0<D[\mathrm{Kpc}]<2.0)$ and stellar fluxes $(3.0<$ $\left.\log L_{*}\left(L_{\odot}\right)<3.6 ; 4.90<\log T_{*}<5.10\right)$.

Very similar results come from the analysis of other "holes" present in NGC 1501, for instance the one at 15 arcsec from the star in $\mathrm{PA}=125^{\circ}$, corresponding to a "window" in the approaching gas, and the one at 11 arcsec from the star in $\mathrm{PA}=-13^{\circ}$, representing a "window" in the receding material (this last hole is visible in Fig. 1 and in Fig. 3 at $\mathrm{PA}=170^{\circ}$, Northern sector).

In spite of the heavy assumptions, the foregoing results would indicate small-scale density fluctuations (combined with ionization) as the main cause of the $N_{\mathrm{e}}$ ([SII]) $N_{\mathrm{e}}(S B)$ discrepancy reported in Sect. 4 .

This implies that:
- the local filling factor in the main shell is $\epsilon_{l}=$ $0.50( \pm 0.1)$, i.e. lower than the value derived from $\left(N_{\mathrm{e}}(S B) / N_{\mathrm{e}}[\mathrm{SII}]\right)^{2}=0.66( \pm 0.1)$;

- our surface brightness electron densities should be increased by a factor $1.4( \pm 0.2)$ to obtain the density peaks of the emitting gas in the main shell.

It is evident that new insights into the variation on a small scale of the physical conditions are needed to deepen our understanding of the above-mentioned questions; this mainly concerns the electron temperature, whose changes affect in different ways the emissivities of recombination and forbidden lines (a lower $T_{\mathrm{e}}$ favouring recombinations; see Peimbert 1994, for a recent review).

As suggested in P1, an even lower value of $\epsilon_{l}$ is expected in the inwards tail of the radial $N_{\mathrm{e}}$ distribution, this knotty and inhomogeneous region arising from hydrodynamical processes.

Moreover, the application of Eq. (1) to the whole nebula gives a global filling factor $\epsilon<0.25$ (corresponding to the parameter normally used to estimate the fraction of the total nebular volume filled by the ionized gas; see Boffi \& Stanghellini 1994, and references therein).

The zooming capability implied in our 3-D analysis is a powerful tool to overcome the existing gap between the potential resolution of the modern photo-ionization 

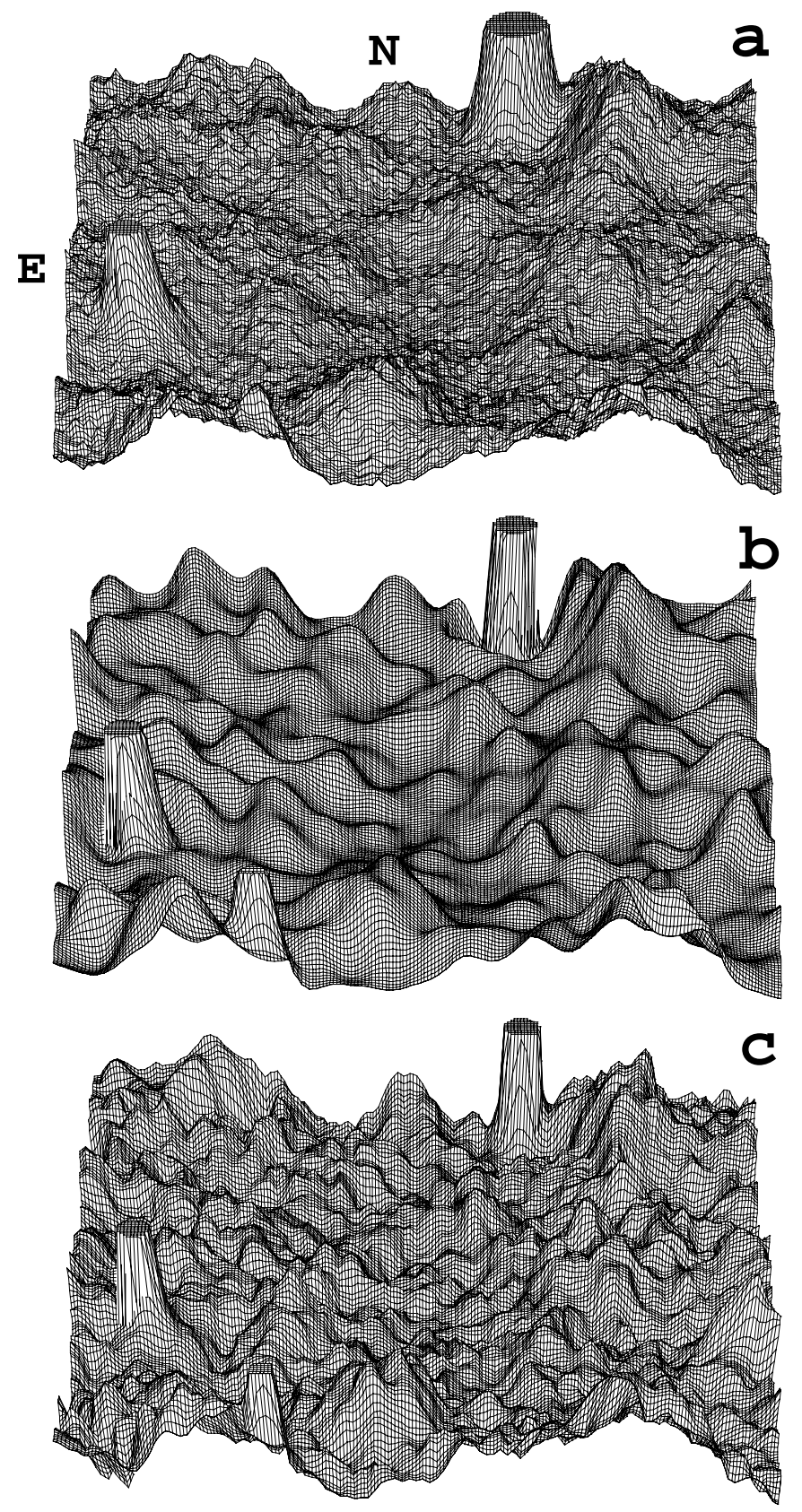

Fig. 10. Flux distributions in the nebular region $(15 \times$ $\left.15 \operatorname{arcsec}^{2}\right)$ containing the "hole" at 11 arcsec from the star in PA $=70^{\circ}$, as obtained from our TNG R frame of NGC 1501 (Fig. 10a), from our TNG R frame after the application of a soft Lucy-Richardson restoration (point spread function $=$ seeing and ten iterations; Fig. 10b), and from the available HST imagery (Fig. 10c; we have assembled the four WFPC2 frames taken by $\mathrm{H}$. Bond). The hole is the roundish region 8 arcsec in diameter at the centre of the $15 \times 15 \operatorname{arcsec}^{2}$ field. The field stars (truncated peaks) projected just outside the hole can be used as identification markers in Fig. 1

models and the actual resolution when applied to real nebulae. The first (for example CLOUDY; Ferland et al. 1998) can be as detailed as one wishes simply using proper input parameters (stellar flux distribution, nebular dimensions, density law etc.), whereas their practical application is limited by observational and reduction procedures and by projection effects: up to now, all efforts intended to isolate a well-defined nebular portion (long-slit spectra analysed at different positions, short-slit echellograms etc.; see Barker 1991, Perinotto \& Corradi 1998 and Hyung et al. 2000 ), in the best cases give emission line intensities which are a mixing of both the approaching gas and receding gas spectra.

The zooming yield is correlated to the spatial resolution of the rebuilt nebula (i.e. to the spatial and spectral resolutions of the echellograms); it is quite modest in the present study of NGC 1501, performed at spatial and spectral resolutions of 1.5-2.5 arcsec and 22000-25000, respectively. The output will increase when analysing the symmetric knots of the quadrupolar PN IC 4634, the ansae and the FLIERS of NGC 7009 (the "Saturn") and the "wings" of the butterfly PN HB 5 (observed with ESO NTT + EMMI at spatial and spectral resolutions of 1.0 arcsec and 60000 , respectively).

\subsection{Radial electron density profile and open questions}

The intersection of the rebuilt-nebula, seen from $(0,0)$, with the plane perpendicular to the line of sight and crossing the central star, gives the $N_{\mathrm{e}}$ map shown in Fig. 11, representing the radial electron density profile (for $\epsilon_{l}=1$ ) extended to all position angles.

Figure 11 shows in detail the most intriguing characteristic of the gas distribution in NGC 1501, i.e. the broad inwards tail present in the radial density profile, probably arising from hydrodynamical processes (Rayleigh-Taylor instabilities and winds interaction; Capriotti 1973; Kahn \& Breitschwerdt 1990; Garcia-Segura et al. 1999).

The first process worked for about $1000 \mathrm{yr}$ in the early evolutionary phases, when the nebula was ionization bounded (up to $R \simeq 0.06 \mathrm{pc}$ ).

The second one, being independent of the optical thickness, acted for much longer (for most of the nebula's life, i.e. $5000 \mathrm{yr}$ ) and is probably still present, as suggested by the large value of $\dot{M}\left(\log \dot{M}=-6.28 M_{\odot} \mathrm{yr}^{-1}\right)$ obtained for the WC4 nucleus of NGC 1501 by Koesterke et al. (1997) using the standard atmosphere model of WolfRayet stars.

This simplified scenario is complicated by the peculiarities of the powering nucleus of NGC 1501 (very hot W-R, rich in $\mathrm{C}$ and $\mathrm{O}$, hydrogen deficient, pulsating), suggesting that the star suffered a thermal pulse in the late postAGB evolution. Its path in the HR diagram is quite uncertain, given the large number of parameters involved: phase in the nuclear burning cycle at which the star departs from the AGB; mass of the residual hydrogen-rich stellar envelope; mass-loss rates during the quiescent hydrogenburning phase, the high-luminosity phase following the helium shell flash and the low-luminosity quiescent helium burning phase; efficiency of convective overshoot in mixing hydrogen-free material out to the stellar surface; chemical 
$\mathrm{N}$

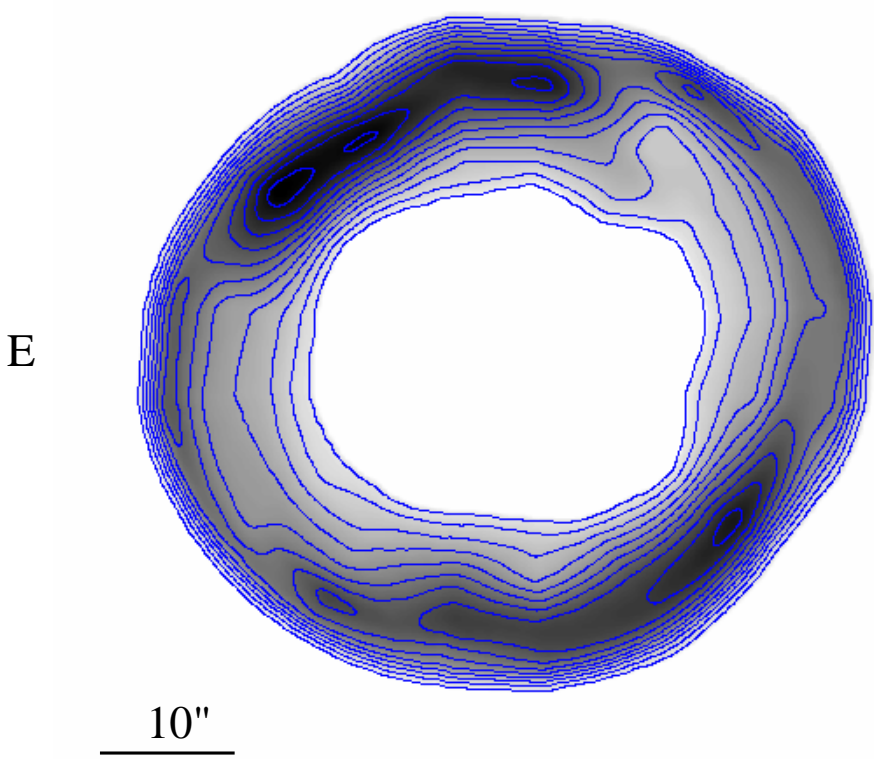

Fig. 11. $N_{\mathrm{e}}$ map and isophotal contours in the sky plane crossing the central star when the nebula is seen from $(0,0)$, showing the peculiar radial density profile of NGC 1501 . The density contours cover the range $200 \mathrm{~cm}^{-3}$ to $1200 \mathrm{~cm}^{-3}$ and are spaced by $100 \mathrm{~cm}^{-3}$. This figure corresponds to the zerovelocity pixel column extended to all position angles (when using high resolution, slit spectroscopy), and to the rest-frame (for Fabry-Perot imagery)

mixing due to stellar rotation (Schönberner 1979; Iben 1984; Blöcker 1995 and references therein).

Thus, the detailed analysis of the mechanisms and physical processes forming and shaping the nebula is deferred to a deeper knowledge of both the star evolutionary parameters and the gas structure.

A final remark concerns the rarity of the radial density profile shown by NGC 1501: no evidence of an extended inward tail is present in the other two dozen PNe up to now spectroscopically observed by us with the Asiago $1.82 \mathrm{~m}$ telescope + Echelle, ESO NTT + EMMI and TNG + SARG.

A good candidate could be NGC 6751; the complex morphology of this nebula (excited by a WC4 star) was shown by Corradi et al. (1996) and spectroscopically studied at high dispersion by Chu et al. (1991), who identified six different components having as many spatio-kinematical characteristics, but didn't give information on the radial density profile of the ionized gas. All this, on the one hand confirms and extends the results already obtained in $\mathrm{P} 1$, and highlights the potentialities of our reconstruction method, on the other hand leaves unresolved many exciting questions, such as:

- what is the small-scale matter distribution in the main shell? And that in the inward tail?
- did the hydrogen-depleted stellar wind create chemical composition gradients across the nebula?

- how large are the electron temperature and turbulence fluctuations in the shell?

- what are the physical conditions, dynamics, ionic and total abundances in the outer, roundish halo?

- is there any observational evidence supporting the "born-again scenario" proposed by Iben et al. (1983; see also Blöcker 1995, and Herwig et al. 1999) for nonradial $g$-mode pulsators?

Most of these (and many other) questions can be answered by a deeper spectroscopic study at higher spatial and spectral resolutions (and at more position angles).

\subsection{Application of the 3-D analysis to expanding nebulae in general}

$\mathrm{PNe}$, nova shells, nebulae ejected by symbiotic stars, bubbles surrounding early spectral type main sequence stars, supernova remnants (SNRs), shells around Population I W-R stars all consist of expanding masses of ionized gas, and the many facets of the 3-D analysis can be successfully applied to them for studying morphology, dynamics, physical conditions, photoionization model and evolutionary status.

In all cases the starting point is high resolution slit spectroscopy (or Fabry-Perot interferometry). Given the large range of expansion velocities shown by the different classes of nebulae (a few dozen $\mathrm{km} \mathrm{s}^{-1}$ for PNe to several hundred $\mathrm{km} \mathrm{s}^{-1}$ for young SNRs and nova shells), the term "high resolution" is here intended relative to $V_{\exp }$.

Thus, the first parameter to be considered is the "relative resolution", $R R=V_{\exp } / \Delta V, \Delta V$ being the spectral resolution.

The best $R R$ value is always a compromise between two contrasting factors, dispersion and detection: a bright nebula can be observed at large $R R$ in both strong and weak lines, whereas only the brightest emissions are detectable in a low surface brightness object (even at small $R R)$.

An indicative lower limit to the applicability of the 3 -D analysis can be put at $R R=3$. This means that slow expanding nebulae (like $\mathrm{PNe}$ ) need high dispersion spectra $(\lambda / \Delta \lambda>20000)$, whereas low dispersion spectroscopy $(\lambda / \Delta \lambda \simeq 1000)$ is preferable for the fastest objects. The latter is clearly illustrated by Lawrence et al. (1995), who derived the three dimensional model of the emitting gas in the Crab Nebula, in Cassiopea A and in the remnant of GK Per (Nova Persei 1901) by means of low resolution $(200<\lambda / \Delta \lambda<1500)$ Fabry-Perot imagery.

A second parameter to be considered in the 3 -D analysis is the spatial resolution along the slit $(\Delta \mathrm{d})$. Also in this case:

- it is intended "relative to the angular dimensions $d$ of the nebula";

- the larger the "relative spatial resolution" $S S=$ $d / \Delta d$, the better the reconstruction; 
- as a rule of thumb, $S S$ must be larger than 3 .

A detailed discussion of the various contributions given by the $3-\mathrm{D}$ analysis in solving the manifold problems connected with the different classes of expanding nebulae is beyond the aims of this paper and is left to the education, experience and imagination of the reader. In fact, our intent is exactly this: to excite the interest of some other people, exposing them to tackle the many problems and the wide applications of the 3-D methodology (we feel quite alone at present... "rari nantes in gurgite vasto" paraphrasing Virgil, Aeneid I, 118).

\section{Conclusions}

This paper introduces a general reduction procedure giving the detailed spatial structure of the ionized gas in expanding nebulae.

Its application to NGC 1501 indicates that this high excitation $\mathrm{PN}$ is an almost oblate ellipsoid of moderate ellipticity $(a \simeq 44 \operatorname{arcsec}, a / b \simeq 1.02, a / c \simeq 1.11)$, denser in the equatorial belt containing the axes $b$ and $c$. A lumpy, quite homogeneous cocoon completely embeds the main shell.

A series of images is presented, showing stereoscopic views of the 3 -D gas structure and the nebular appearance when changing the line of sight.

The morphology-structure correlation is discussed, the small scale density variations in the main shell and the peculiarities of the matter radial profile are analysed.

NGC 1501 is a quite faint, high ionization, density bounded PN, its 3-D study is simple and straightforward (at our spatial and spectral resolutions).

We are now involved in a much more ambitious project: the spatial mapping in different ions $\left(\mathrm{H}^{+}, \mathrm{He}^{+}, \mathrm{He}^{++}\right.$, $\mathrm{O}^{\circ}, \mathrm{O}^{+}, \mathrm{O}^{++}, \mathrm{N}^{+}, \mathrm{S}^{+}, \mathrm{Ne}^{++}, \mathrm{Ar}^{++}, \mathrm{Ar}^{+++}$etc.) and the photo-ionization modelling of a dozen bright $\mathrm{PNe}$ in both hemispheres; each target observed at several (at least 9) position angles with ESO NTT + EMMI (spatial resolution $=1.0$ arcsec, spectral resolution $=60000)$ and $\mathrm{TNG}+\mathrm{SARG}$ (spatial resolution $=0.7$ arcsec, spectral resolution $=115000)$.

Acknowledgements. We greatly appreciated the suggestions and the encouragement by Luciana Bianchi, Gary Ferland, Guillermo Garcia-Segura and Vincent Icke; to them all we express our deep gratitude.

This paper is based on observations made with:

- the $1.82 \mathrm{~m}$ telescope of the Astronomical Observatory of Padua, operated at Asiago, Cima Ekar (Italy);

- the Italian Telescopio Nazionale Galileo (TNG) operated on the island of La Palma by the Centro Galileo Galilei of the CNAA (Consorzio Nazionale per l'Astronomia e l'Astrofisica) at the Spanish Observatorio del Roque de los Muchachos of the Instituto de Astrofisica de Canarias;

- the NASA/ESA Hubble Space Telescope, obtained from the data archive at the Space Telescope Science Institute. STScI is operated by the Association of Universities for Research in Astronomy, Inc. under NASA contract NAS 5-26555.

\section{References}

Acker, A. 1978, A\&A, 33,367

Acker, A., Ochsenbein, F., Stenholm, B., et al. 1992, Strasbourg-ESO Catalogue of Galactic Planetary Nebulae, ESO, Garching

Acker, A., Raytchev, B., Stenholm, B., \& Tylenda, R. 1991, A\&AS, 90, 89

Aller, L. H. 1976, MSRSL, 9, 271

Aller, L. H., \& Czyzak, S. J. 1983, ApJS, 51, 211

Aller, L. H., \& Epps, H. W. 1976, ApJ, 204, 445

Amnuel, P. R., Guseinov, O. H., Novruzova, H. I., \& Rustamov, Y. S. 1984, Ap\&SS, 107, 19

Atherton, P. D., Hicks, T. R., Reay, N. K., Worswick, S. P., \& Smith, W. H. 1978, A\&A, 66, 297

Bachiller, R., Bujarrabal, V., Martin-Pintado, J., \& Gomez-Gonzalez, J. 1989, A\&A, 218, 252

Baker, J. G., \& Menzel, D. H. 1938, ApJ, 88, 52

Balick, B. 1987, AJ, 94, 671

Balick, B., Gonzales, G., Frank, A., \& Jacoby, G. 1992, ApJ, 392, 582

Barker, T. 1991, ApJ, 371, 217

Blöcker, T. 1995, A\&A, 299, 755

Boffi, F. R., \& Stanghellini, L. 1994, A\&A, 284, 248

Bond, H. E., Kawaler, S. D., Ciardullo, R., et al. 1996, AJ, 112,2699

Brocklehurst, M. 1971, MNRAS, 153, 471

Bryce, M., Balick, B., \& Meaburn, J. 1994, MNRAS, 266, 721

Cahn, J. H. 1976, AJ, 81, 407

Cahn, J. H., \& Kaler, J. B. 1971, ApJS, 22,319

Cahn, J. H., Kaler, J. B., \& Stanghellini, L. 1992, A\&AS, 94, 399

Capriotti, E. R. 1973, ApJ, 179, 495

Chu, Y.-H., Jacoby, G., \& Arendt, R. 1987, ApJS, 64, 529

Chu, Y.-H., Manchado, A., Jacoby, G. H., \& Kwitter, K. B. 1991, ApJ, 376, 150

Ciardullo, R., \& Bond, H. E. 1998, AJ, 111, 2332

Ciardullo, R., Bond, H. E., Sipior, M. S., et al. 1999, AJ, 118, 488

Collins, G. W., Daub, C. T., \& O’Dell, C. R. 1961, ApJ, 133, 471

Corradi, R. L. M., Manso, R., Mampaso, A., \& Schwarz, H. E. 1996, A\&A, 313, 913

Corradi, R. L. M., \& Schwarz, H. E. 1995, A\&A, 293, 871

Cudworth, K. M. 1974, AJ, 79, 1384

Curtis, H. D. 1918, Publ. Lick Obs., 13, 55

Daub, C. T. 1982, ApJ, 260, 612

Dwarkadas, V. V., \& Balick, B. 1998, ApJ, 497, 267

Feibelman, W. A. 1998, ApJS, 119, 197

Feibelman, W. A. 2000, ApJ, 542, 957

Ferland, G. J., Korista, K. T., Verner, D. A., et al. 1998, PASP, 110,761

Garcia-Segura, G., Langer, N., Rozyczka, M., \& Franco, J. 1999, ApJ, 517, 767

Gorny, S. K., Schwarz, H. E., Corradi, R. L. M., \& Van Winckel, H. 1999, A\&AS, 136, 145

Greig, W. E. 1972, A\&A, 18, 70

Guerrero, M. A., Manchado, A., \& Chu, Y.-H. 1997, ApJ, 487, 328

Guerrero, M. A., Manchado, A., \& Serra-Ricart, M. 1996, ApJ, 456,651

Habing, H. J. 1996, A\&AR, 7, 97 
Hajian, A. R., Terzian, Y., \& Bignell, C. 1993, AJ, 106, 1965 Hajian, A. R., Terzian, Y., \& Bignell, C. 1995, AJ, 109, 2600

Hamann, W.-R. 1997, in IAU Symp. 180, Planetary Nebulae, ed. H. J. Habing, \& H. J. G. L. M. Lamers (Kluwer), 91

Herwig, F., Blöcker, T., Langen, N., \& Driebe, T. 1999, A\&A, 349, L5

Hua, C. T., \& Louise, R. 1970, A\&A, 9, 448

Hyung, S., Aller, L. H., Feibelman, W. A., \& de Koter, A. 2000, MNRAS, 318, 77

Iben, I. 1984, ApJ, 277, 333

Iben, I. Jr., Kaler, J. B., Truran, J. W., \& Renzini, A. 1983, ApJ, 264, 605

Kahn, F. D., \& Breitschwerdt, D. 1990, MNRAS, 242, 505

Kaler, J. B. 1976, ApJS, 31, 517

Kawamura, J., \& Masson, C. 1996, ApJ, 461, 282

Koesterke, L., \& Hamann, W.-R. 1997, in IAU Symp. 180, Planetary Nebulae, ed. H. J. Habing, \& H. J. G. L. M. Lamers (Kluwer), 114

Kupferman, P. N. 1983, ApJ, 266, 689

Lame, N. J., \& Pogge, R. W. 1994, AJ, 108, 1860

Lawrence, S. S., MacAlpine, G. M., Uomoto, A., et al. 1995, AJ, 109, 2635

Louise, R. 1974, A\&A, 30, 189

Maciel, W. J. 1984, A\&AS, 55, 253

Maciel, W. J., \& Quireza, C. 1999, A\&A, 345, 629

Manchado, A., Guerrero, M. A., Stanghellini, L., \& Serra-Ricart, M. 1996, The IAC Morphological Catalog of Northern Planetary Nebulae, IAC

Masson, C. R. 1990, ApJ, 348, 580

Mellema, G. 1997, A\&A, 321, L29

Minkowski, R. 1968, in IAU Symp. 34, ed. D. E. Osterbrock, \& C. R. O'Dell (Dordrecht-Reidel), 456

Minkowski, R., \& Osterbrock, D. 1960, ApJ, 131, 537

Morris, M. 1987, PASP, 99, 115

Neiner, C., Acker, A., Gesicki, K., \& Szczerba, R. 2000, A\&A, 358,321

O'Dell, C. R. 1962, ApJ, 135, 371

Page, T. L. 1942, ApJ, 96, 78

Pascoli, G. 1990, A\&A, 232, 148

Pascoli, G. 1992, PASP, 104, 305

Pease, F. G. 1917, ApJ, 46, 42

Peimbert, M. 1994, The Analysis of Emission Lines, ed. R. Williams, \& M. Livio (Cambridge Univ. Press, Cambridge), 165
Peimbert, M., \& Serrano, A. 1980, Rev Mex. Astron. Astrofis., 5,9

Perinotto, M., \& Corradi, R. L. M. 1998, A\&A, 332, 721

Phillips, J. P., \& Reay, N. K. 1980, ApJL, 21, 47

Pottasch, S. R. 1983, in Planetary Nebulae, a Study of Late Stages of Stellar Evolution (Dordrecht-Reidel), 105

Proisy, P. E. 1974, A\&A, 35, 71

Rauch, T. 1999, A\&AS, 135, 487

Reay, N. K., \& Worswick, S. P. 1977, MNRAS, 179, 317

Robinson, G. J., Reay, N. K., \& Atherton, P. D. 1982, MNRAS, 199,649

Sabbadin, F. 1984, MNRAS, 210, 341

Sabbadin, F. 1986, A\&A, 160, 31

Sabbadin, F., Benetti, S., Cappellaro, E., \& Turatto, M. 2000b, A\&A, 361, 1112 (P1)

Sabbadin, F., Bianchini, A., Ortolani, S., \& Strafella, F. 1985, MNRAS, 217, 539

Sabbadin, F., Cappellaro, E., Benetti, S., Turatto, M., \& Zanin, C. 2000a, A\&A, 355, 688

Sabbadin, F., Cappellaro, E., \& Turatto, M. 1987, A\&A, 182, 305

Sabbadin, F., \& Hamzaoglu, E. 1982, A\&AS, 50, 1

Schönberner, D. 1979, A\&A, 79, 108

Schönberner, D. 1997, in IAU Symp. 180, Planetary Nebulae, ed. H. J. Habing, \& H. J. G. L. M. Lamers (Kluwer), 379

Schwarz, H. E., Corradi, R. L. M., \& Melnick, J. 1992, A\&AS, 96,23

Stanghellini, L., Corradi, R. L. M., \& Schwarz, H. E. 1993, A\&A, 279, 521

Stanghellini, L., Kaler, J. B., \& Shaw, R. A. 1994, A\&A, 291, 604

Swings, P., \& Swensson, J. W. 1942, AnnAp, 15, 290

Terzian, Y. 1997, in IAU Symp. 180, Planetary Nebulae, ed. H. J. Habing, \& H. J. G. L. M. Lamers (Kluwer), 29

Tylenda, R. 1986, A\&A, 156, 217

Tylenda, R., Acker, A., Raytchev, B., Stenholm, B., \& Gleizes, F. 1991, A\&AS, 89, 77

Tylenda, R., Acker, A., \& Stenholm, B. 1993, A\&AS, 102, 595

Van de Steene, G. C., \& Zijlstra, A. A. 1994, A\&AS, 108, 485

Vassiliadis, E., \& Wood, P. R. 1993, ApJ, 413, 641

Volk, K., \& Leahy, D. A. 1993, AJ, 106, 1954

Zhang, C. Y. 1995, ApJS, 98, 659

Zuckerman, B., \& Aller, L. H. 1986, ApJ, 301, 772 